\title{
Genome-wide identification and characterization of InDels and SNPs in Glycine max and Glycine soja for contrasting seed permeability traits
}

G. Ramakrishna ${ }^{1 \dagger}$, Parampreet Kaur ${ }^{1 \dagger}$, Deepti Nigam ${ }^{1 \dagger}$, Pavan K. Chaduvula', Sangita Yadav², Akshay Talukdar ${ }^{3}$, Nagendra Kumar Singh ${ }^{1}$ and Kishor Gaikwad ${ }^{1 *}$

\begin{abstract}
Background: Water permeability governed by seed coat is a major facet of seed crops, especially soybean, whose seeds lack physiological dormancy and experience rapid deterioration in seed viability under prolonged storage. Moreover, the physiological and chemical characteristics of soybean seeds are known to vary with seed coat color. Thus, to underpin the genes controlling water permeability in soybean seeds, we carried out an in-depth characterization of the associated genomic variation.

Results: In the present study, we have analyzed genomic variation between cultivated soybean and its wild progenitor with implications on seed permeability, a trait related to seed storability. Whole genome resequencing of G.max and G. soja, identified SNPs and InDels which were further characterized on the basis of their genomic location and impact on gene expression. Chromosomal density distribution of the variation was assessed across the genome and genes carrying SNPs and InDels were characterized into different metabolic pathways. Seed hardiness is a complex trait that is affected by the allelic constitution of a genetic locus as well as by a tricky web of plant hormone interactions. Seven genes that hold a probable role in the determination of seed permeability were selected and their expression differences at different stages of water imbibition were analyzed. Variant interaction network derived 205 downstream interacting partners of 7 genes confirmed their role in seed related traits. Interestingly, genes encoding for Type I- Inositol polyphosphate 5 phosphatase1 and E3 Ubiquitin ligase could differentiate parental genotypes, revealed protein conformational deformations and were found to segregate among RILs in coherence with their permeability scores. The 2 identified genes, thus showed a preliminary association with the desirable permeability characteristics.
\end{abstract}

Conclusion: In the light of above outcomes, 2 genes were identified that revealed preliminary, but a relevant association with soybean seed permeability trait and hence could serve as a primary material for understanding the molecular pathways controlling seed permeability traits in soybean.

Keywords: Seed permeability, NextGen sequencing, Soybean, SNPs, InDels

\footnotetext{
*Correspondence: kish2012@nrcpb.org

${ }^{\dagger} \mathrm{G}$. Ramakrishna, Parampreet Kaur and Deepti Nigam contributed equally to this work.

'ICAR- National Research Centre on Plant Biotechnology, Pusa Campus, New

Delhi 110012, India

Full list of author information is available at the end of the article
}

(c) The Author(s). 2018 Open Access This article is distributed under the terms of the Creative Commons Attribution 4.0 International License (http://creativecommons.org/licenses/by/4.0/), which permits unrestricted use, distribution, and reproduction in any medium, provided you give appropriate credit to the original author(s) and the source, provide a link to the Creative Commons license, and indicate if changes were made. The Creative Commons Public Domain Dedication waiver (http://creativecommons.org/publicdomain/zero/1.0/) applies to the data made available in this article, unless otherwise stated. 


\section{Background}

Soybean is cultivated for two economically and nutritionally important compounds, i.e., protein and oil, which together constitute approximately $56 \%$ of its dry seed weight. Wild (G. soja) and cultivated soybean (G. max) differ in various morphological and physiological characteristics. Large seeds with variable seed coat colors are characteristic of cultivated varieties and referred to as permeable seeds, whereas wild species possess small, coarse black and hard seeds that display water impermeability. Wild and cultivated soybean differ in the extent of hardseededness, though considerable variation exists in the later for water permeability [1-3]. Hardseededness has a multitude of effects that raises both biological and economic concerns as it enables perseverance of seed stocks for several years [4] by providing resistance and protection from seed spoilage and seed pathogens but poses a major problem for seed germination that is required to generate high crop yields $[4,5]$. Thus, moderately impermeable seed cultivars are desirable as they can maintain their post-harvest viability and quality.

Both environmental and genetic factors contribute towards soybean seed permeability. Morphologically, hardseededness is related to the absence of cracks in seed coat [6]. Complex multilayer seed coat and its chemical constituents are crucial determinants of a water barrier system of the seed coat, and presence of phenolic compounds has also been associated with seed coat impermeability [2, 7]. Numerous studies have associated insoluble lipid polyesters (cutin and cutin like depositions) in seed coat of Arabidopsis [8], brassica [9] and soybean [10] with seed impermeability. In Arabidopsis, mutations in genes such as LAC15 [11], acyl-CoA:glycerol3-phosphate acyltransferase, suberin biosynthetic gene GPAT5 [12] etc., has led to higher permeability than the wild-type seeds. Phytohormones such as abscisic acid (ABA) also play an important role in the regulation of seed germination [13-15] and triggers ABI5 accumulation and phosphorylation to repress germination [16]. In Arabidopsis [17] and Medicago [18], ABI5 is identified as a prominent regulator of seed maturation and longevity. To understand the genetic basis of seed hardiness, different studies have reported the presence of a common QTL on an overlapping region of soybean chromosome 2 [19-22]. Sun et al. has delimited this QTL to a $22 \mathrm{~Kb}$ region containing 2 genes, of which SNP $(\mathrm{C}>\mathrm{T})$ in the 8th exon of Glyma02g43700.1, designated as GmHs1-1 and encoding for Calcineurin like metallophosphoesterase transmembrane protein in malphigian layers of seed coat, could effectively distinguish between parental genotypes and 8 additional G. soja accessions [23]. Another locus positioned closely to GmHs1-1 is qHS1QTL, which encodes for endo-1,4-ßglucanases and results in accumulation of $\beta 1$ - 4glucan derivatives that reinforces hardseededness in soybean [24].

In the present study, we have attempted to identify variation (SNPs and InDels) between genomes of $G$. $\max$ and G. soja in relation to their seed permeability characteristics. SNPs and InDels were categorized into different metabolic pathways and genes were selected from pathways that could influence seed permeability, followed by their validation through real-time expression studies. Using In-silico approaches, various downstream analysis were conducted for candidate genes to reveal an association between their structure and permeability. Further, we have shown that genomic variation in the selected genes could be further developed into markers to distinguish permeable and impermeable parental genotypes and their RILs in coherence with their permeability scores. Although the numbers of SSR and RFLP markers are known to be associated with seed permeability and hardseededness, this is the primary but comprehensive report on identification and association of genome-wide SNP and InDels with seed permeability.

\section{Results}

Resequencing, mapping, and assembly of G. max and G. soja

Workflow diagram of the analysis done is shown in Fig. 1. A total of 118.8 million and 119.8 million potentially paired-end reads of G. max and G. soja were obtained respectively. Sequence information has been deposited in the BioProject database of NCBI, under ID PRJNA383915. A total of $117.11 \mathrm{M}(98.57 \%)$ and $113.66 \mathrm{M}$ (94.87\%) high quality filtered reads of G. $\max$ and G. soja were successfully mapped to the reference genome (G. max var. Williams 82) with a coverage of 12.56 and $12.32 \mathrm{X}$, respectively (Additional file 1: Table $\mathrm{S} 1$ ).

\section{Identification and chromosomal distribution of SNPs and InDels}

A total of 77,339 and 215932 SNPs as well as 451,522 and 697,295 InDels were identified in G. $\max$ and G. soja, respectively, upon comparison with the reference genome after filtering. The average density of SNPs and InDels in G. $\max$ was observed to be about $79.04 / \mathrm{Mb}$ and $461.48 / \mathrm{Mb}$ and 291.01 SNPs/Mb and 712.67 InDels/Mb in G. soja. In total, 10,873 SNPs and 80,078 InDels were found to be common to both species. A total of 40,130 SNPs and 381,644 InDels of G. max could be mapped onto 7903 and 32,116 genes, respectively, while in G. soja 154,611 SNPs and 595,433 InDels were mapped onto 29,823 and 51,310 genes, respectively. A total of 1760 genes in G. $\max$ (Additional file 2) and 29,530 genes in G. soja (Additional files 3 and 4) were found to possess both SNPs and InDels. 


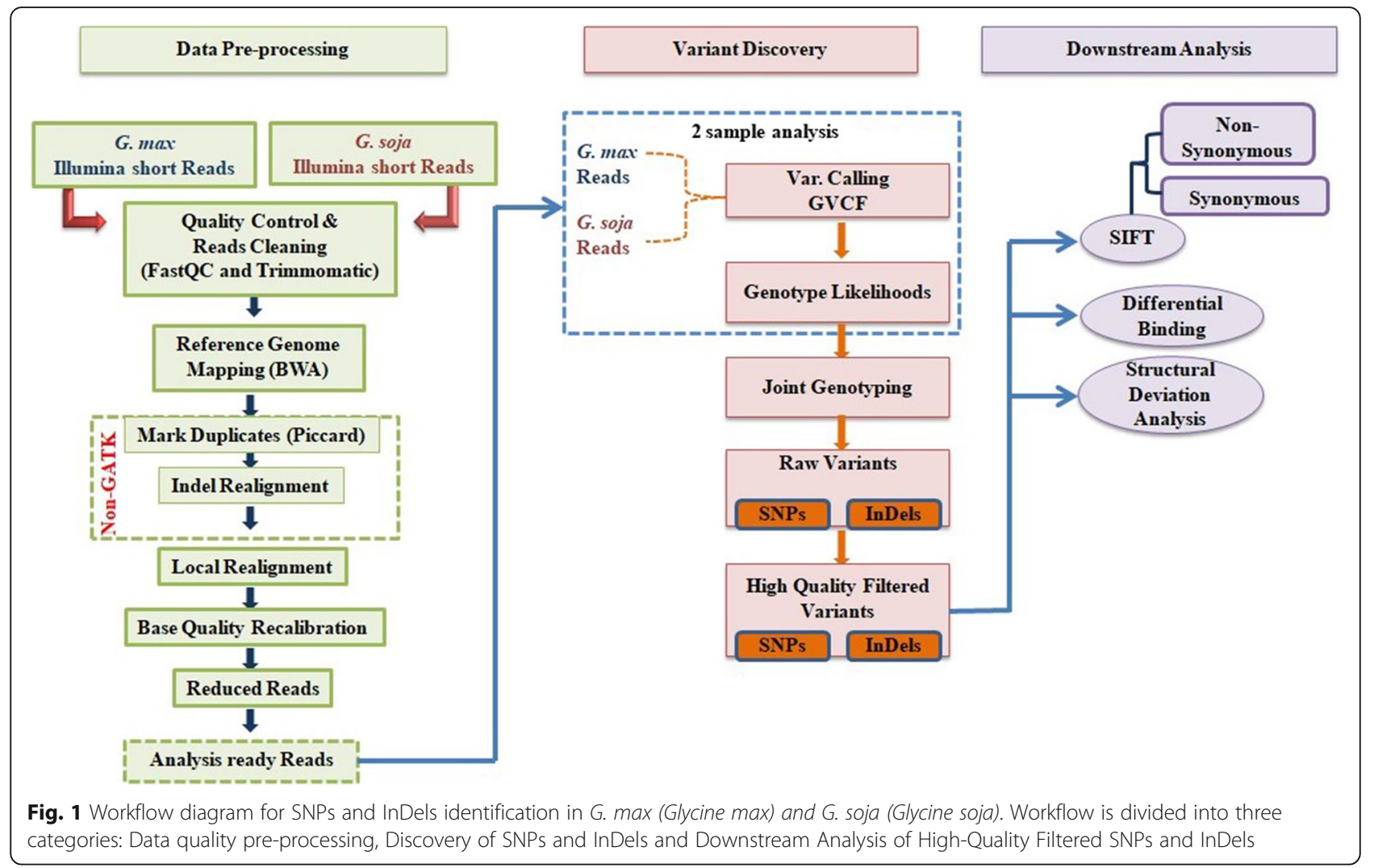

Numbers of transition (Ts) and transversion (Tv) SNPs identified in G. $\max$ have shown $\mathrm{Ts} / \mathrm{Tv}$ ratio of 1.814 and 1.859 in. G. soja $\mathrm{A} / \mathrm{T}$ and $\mathrm{G} / \mathrm{T}$ represented most frequent transversion while frequencies of $\mathrm{C} / \mathrm{T}$ and $\mathrm{A} / \mathrm{G}$ transitions were similar in both species. Ts/Tv ratio of 2.81, 2.65 and 3.41 was observed at first, second and third codon position, respectively. Deletions and insertions of length observed in G. max ranged from 1 to $90 \mathrm{bp}$ and 1 to $75 \mathrm{bp}$, respectively, while a range of 1 to $110 \mathrm{bp}$ and 1 to $80 \mathrm{bp}$, respectively was observed for G. soja.

In both species, non-uniform distribution of SNP and InDels across 20 chromosomes was observed (Fig. 2a). Chromosome 18 is the largest chromosome and possessed the highest number of InDels and SNPs in G. $\max$ i.e., 670.47 InDels/Mb and 165.18 SNPs/Mb. While the least number of InDels (221.77 InDels/Mb) and SNPs (24.66 SNPs/Mb) were mapped on chromosome 20 and chromosome 4 of G. max, respectively. Chromosome 8 of G. soja, possessed a maximum number of InDels (951.23 InDels/Mb) and SNPs (322.86 SNP/Mb) across its genome. The least number of SNPs and InDels were mapped onto chromosome 9 (108.02 SNPs/Mb) and chromosome 11 (855.30 InDels/Mb) of G. soja, respectively. Further, for both species, most dense distribution of variation towards the chromosome ends was observed (Fig. 2b). Less InDels were detected with increasing length while different InDels of the same length shared similar abundance in both species.

\section{Classification and categorization of SNPs and InDels}

SNPs and InDels were further categorized into various groups (Table 1). InDels and SNPs were more abundantly observed in upstream and downstream regulatory regions (URR and DRR, respectively) of genes. A total of 1876 InDels resulted in translation frame-shifts, while 1227 InDels caused deletion/insertion of amino acids in G. max whereas in G. soja the number ranged from 2287 and 1892 InDels, respectively. A total of 60 and 219 SNPs in G. max and G. soja, respectively, were found to affect essential splice donor or acceptor sites. Impact of variation in both species collectively was categorized as: low impact $(0.22 \%)$, modifier $(99.08 \%)$, moderate $(0.35 \%)$, and high impact variation $(0.34 \%)$. Overall, a high frequency of SNPs and InDels was observed to influence the gene function in G. soja relative to G. $\max$. In both G. $\max$ and G. soja, low impact SNPs were higher in number compared to that of high impact SNPs, but a reverse trend was observed for low and high impact InDels. Collectively, 125 high impact SNPs and 4594 InDels were identified in both species which affected 1459 and 1839 genes in G. $\max$ and G. soja, respectively. Cumulatively, in G. $\max$ and G. soja, 45 and 83 high impact SNPs and InDels, 


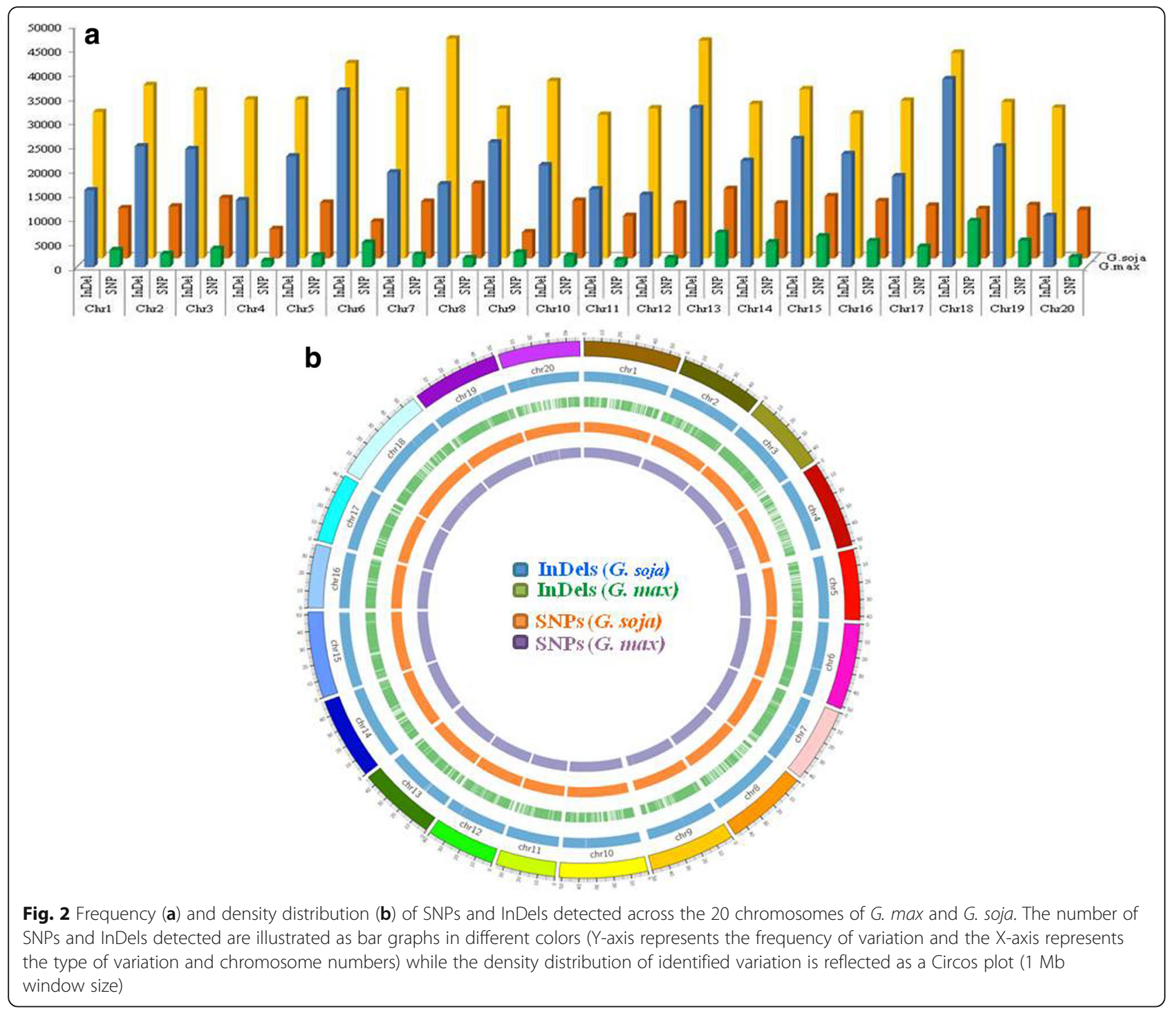

Table 1 Categorization of SNPs and InDels in G. max and G. soja (URR: Upstream regulatory regions, DRR: Downstream regulatory regions, CDS: Coding sequences)

\begin{tabular}{|c|c|c|c|c|}
\hline Variations & Type of Variation & & Frequency in G. max & Frequency in G. sojo \\
\hline \multirow[t]{5}{*}{ SNPS } & Gene Based & URR & 11,508 & 73,384 \\
\hline & & DRR & 9546 & 60,406 \\
\hline & & CDS & 4386 & 20,831 \\
\hline & Intergenic & & 925 & 31,936 \\
\hline & Non-synonymous & & 380 & 903 \\
\hline \multirow[t]{5}{*}{ InDels } & Gene Based & URR & 153,820 & 265,741 \\
\hline & & DRR & 133,212 & 228,455 \\
\hline & & CDS & 62,335 & 101,156 \\
\hline & Intergenic & & 65,090 & 101,823 \\
\hline & Non-synonymous & & 1077 & 2013 \\
\hline
\end{tabular}


respectively, were identified to result in a gain of stop codon while 22 and 40 high impact SNPs and InDels, respectively accounted for the loss of stop codon in a transcript. Approximately, 90 and $75 \%$ of the low impact SNPs represented synonymous SNPs in G. max and G. soja, respectively, wherein an amino acid encoded by a gene remained unaltered thus imparting low impact on gene functionality. Modifier SNPs were mostly represented in upstream and downstream gene variants in both G. $\max$ and G. soja. Approximately, $82 \%$ of the high impact InDels resulted in frameshift variation in G. max and G. soja while the low impact InDels were present in intron regions and splice site regions. Further, 70 and 148 SNPs in G. $\max$ and G. soja, respectively, were found to possess SIFT score $\leq$ -2.5 and were termed as deleterious. Among non-synonymous variants, 69 and 143 SNPs in G. max and G. soja, respectively, were deleterious.

Further, non-synonymous SNPs and InDels were mapped uniquely onto 76 pathways in G. $\max$ and 102 pathways in G. soja. Interestingly, 9 and 35 pathways were unique to G. max and G. soja, respectively, whereas 67 pathways were found to be common between them. This classification identified a diverse array of pathways ranging from cellular metabolism, transcriptional regulators for genes functioning under stress conditions as well as defense pathways (Additional file 5).

\section{Analysis of differential binding motif and variant interaction network (VIN) reveals new players governing seed permeability differences}

Seven genes carrying non-synonymous variants having deleterious effects on gene function were found to be involved in seed permeability related metabolic pathways (Table 2). The variable region of these genes accounted for differential binding of 13 transcription factor (TF) motifs between G. soja and G. $\max$ (Fig. 3a). The higher occurrence of motifs for AP2, Myb/SANT, Homeodomain, SBP, TCP, AT hook, CxC, GATA, C2H2 ZF, B3, MADF, and CG-1 were observed in G. soja in comparison to G. max. Interestingly, except Myb/SANT and homeodomain motif, other 10 motifs were observed as unique to the G. soja.

To further explore the possible effect of variation identified in the context of their role in mediating differential TF's binding events, interaction network of 7 selected genes (guide genes) was constructed that identified 205 interacting partners distributed in 2 network communities (Fig. 3b). The community I was regulated by 1 guide gene, i.e., Phospholipase D (Glyma20g38200) and was enriched for TFs with AT-hook and $\mathrm{CxC}$ motif, while community II was regulated by 4 guide genes viz. Glycosyltransferases (Glyma13g05441), Chalcone flavone isomerase (Glyma03g31100), ABI5 (Glyma19g37910) and Type I/Type I/InsP 5-ptase (Glyma02g08620) and these hub genes were enriched with differential binding motifs for AP2, Myb/SANT, Homeodomain, SBP, TCP, B3, CG-1, AP2, B3 (as complex) and GATA TFs, wherein AP2, Myb/SANT, Homeodomain, SBP were represented in at least 2 guide genes. Further, these 205 downstream interacting partners were annotated and classified into biological processes, molecular functions and cellular components (Additional file 6: Figure S1, Additional file 7: Figure S2, Additional file 8: Figure S3; Additional file 9), with the majority of annotations specific to seed related traits.

\section{DNA polymorphism and real-time expression studies}

DNA polymorphism observed between parental genotypes for all genes was in accordance to in silico

Table 2 Features of genes involved in seed coat permeability selected for further analysis (Chr. No. - Chromosome Number; bp - base pairs)

\begin{tabular}{|c|c|c|c|c|c|}
\hline Gene (Glyma Ids) & $\begin{array}{l}\text { Gene } \\
\text { length } \\
(\mathrm{bp})\end{array}$ & $\begin{array}{l}\text { Variation position (Chr No.: } \\
\text { start position coordinates) }\end{array}$ & $\begin{array}{l}\text { Variation identified } \\
\text { (G.max / G.soja) }\end{array}$ & $\begin{array}{l}\text { Variation effect (G. max/G. soja } \\
\text { amino acid change) on protein }\end{array}$ & $\begin{array}{l}\text { Impact of } \\
\text { variability }\end{array}$ \\
\hline $\begin{array}{l}\text { Anthranilate } n \text {-hydroxy cinnamoyl/ } \\
\text { benzoyl transferase (Glyma17g16330) }\end{array}$ & 4249 & 17:12977991 & $---/ A A A$ & $(-/ K)$ & Moderate \\
\hline $\begin{array}{l}\text { Chalcone flavone isomerase } \\
\text { (Glyma03g31100) }\end{array}$ & 6050 & 3:38990976 & $\begin{array}{l}---------/ A C G G \\
\text { CCACG }\end{array}$ & $(-N A V)$ & Moderate \\
\hline $\begin{array}{l}\text { Abscisic Acid Insensitive } 5 \\
\text { (Glyma19g37910) }\end{array}$ & 5266 & 19:45001036 & $---/ G G T$ & $(-/ G)$ & Moderate \\
\hline PhospholipaseD (Glyma20g38200) & 10,132 & $20: 45855223$ & $---/ T \mathrm{TG}$ & $(-/ L)$ & Moderate \\
\hline $\begin{array}{l}\text { Typel- Inositol polyphasphate } 5 \\
\text { phosphotase1 (Glyma02g08620) }\end{array}$ & 7867 & 2:6711434 & $\begin{array}{l}---------- \\
-/ \text { GGACAACAGGCA }\end{array}$ & $(-/ D N R C)$ & Moderate \\
\hline E3 ubiquitin ligase (Glyma10g43120) & 4938 & 10:49932855 & $\begin{array}{l}----------/ T A T A \\
\text { GATATA }\end{array}$ & $\begin{array}{l}\text { Stop codon gain, Protein } \\
\text { truncation }\left(P / L^{*} \mid Y X\right)\end{array}$ & High \\
\hline Glycosyl transferase (Glyma13g05441) & 5969 & 13:5763533 & $\begin{array}{l}--/ T A \text { (Insertion is } \\
\text { present upstream to } \\
\text { gene) }\end{array}$ & - & Moderate \\
\hline
\end{tabular}




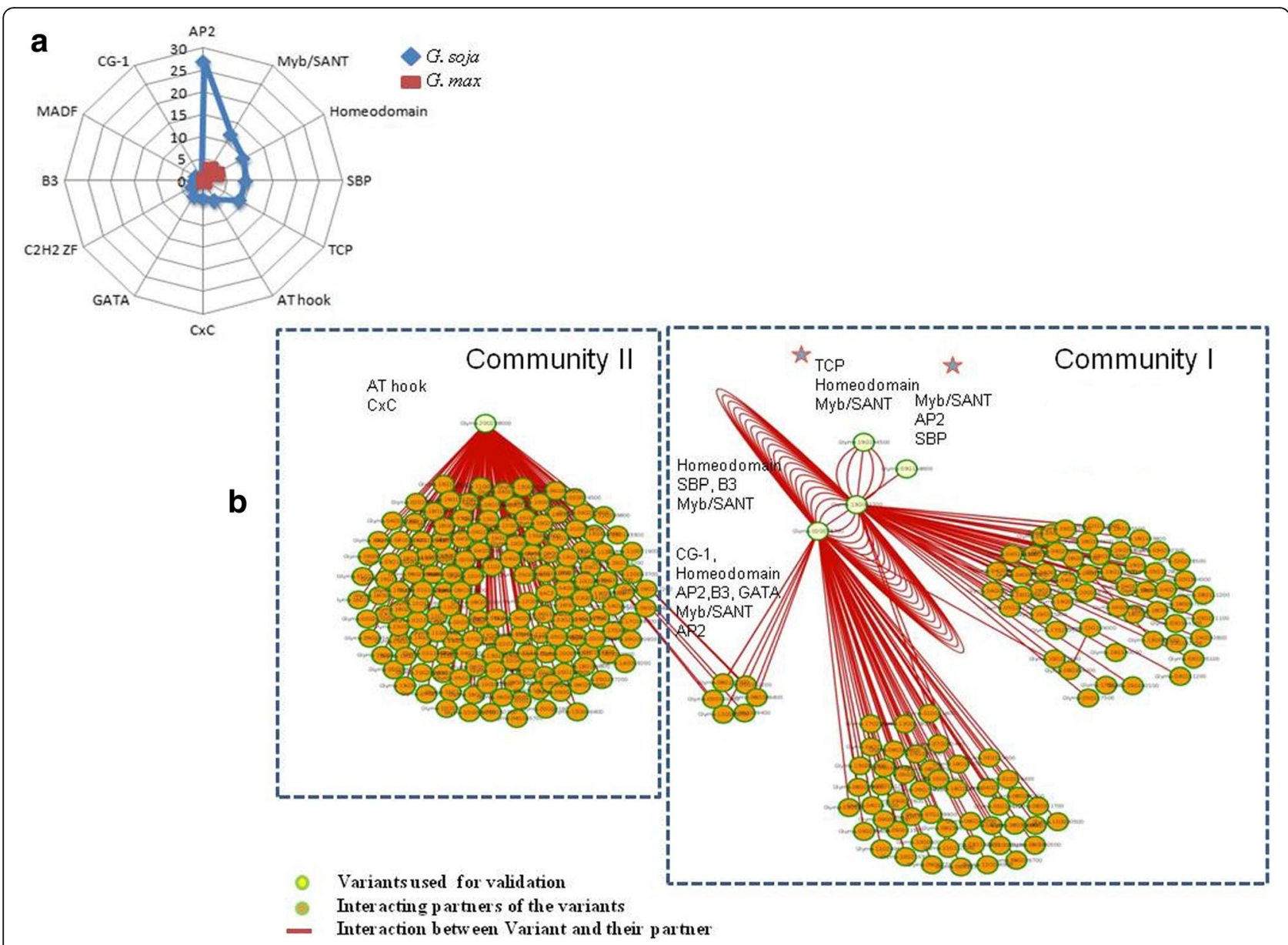

Fig. 3 Frequency of differential binding motifs identified at variation position of 7 genes between G. max and G. soja (Numeric indicating motif frequency) (a); Variant Interaction Network (VIN) representing the interacting partners for seven genes in two major communities (b)

predictions (Additional file 10: Figure S4). Except for gene Type I Inositol Polyphosphate 5 Phosphatase1 (Type I/InsP 5-ptase), for which no efficient real-time primers could be designed, another 6 genes were analyzed for differences in transcript abundance at 5 different time intervals of water imbibition of the seed (Fig. 4).

The number of transcripts of Glycosyltransferases were found to increase during imbibition, with 2.5 fold increase at $72 \mathrm{~h}$ in comparison to control whereas $G$. soja transcripts were relatively less abundant, though a transient increase was observed in comparison to $G$. $\max$ at $24 \mathrm{~h}$ of imbibition. A slow increase in transcript level of Phospholipase D occurred in G. max in contrast to G. soja. Maximum number of transcripts were present at $24 \mathrm{~h}$ of imbibition in G. soja followed by a decline thereafter. A gradual decline in the transcript level of Anthranilate n-hydroxy cinnamoyl/benzoyl transferase (HCBT) was observed in G. soja with a transient increase at $48 \mathrm{~h}$ of imbibition. In G. $\max$, HCBT transcripts were present at low levels during imbibition in comparison to control seeds, though an increase in transcript number was observed from 12 to $48 \mathrm{~h}$ of imbibition followed by a dip thereafter. Transcripts of Abscisic acid insensitive 5 (ABI5) were present in lower amounts in both G. max and G. soja during imbibition in comparison to that of control seeds. An antagonistic pattern of ABI5 transcript abundance was observed between G. max and G. soja. A 2 fold increase observed in transcript level of Chalcone flavone isomerase in G. $\max$ was in contrast to 2 fold reduction observed for transcripts in G. soja until $24 \mathrm{~h}$ of imbibition. This was followed by a decline in transcripts of G. $\max$ and increased expression in G. soja at $48 \mathrm{~h}$ of imbibition. The transcript level of E3 Ubiquitin ligase did not reveal any particular pattern of abundance, though the transcript level increased gradually from 24 to $48 \mathrm{~h}$ followed by a dip thereafter in G. soja, an antagonistic expression pattern was observed in G. $\max$ and G. soja with respect to each other. 

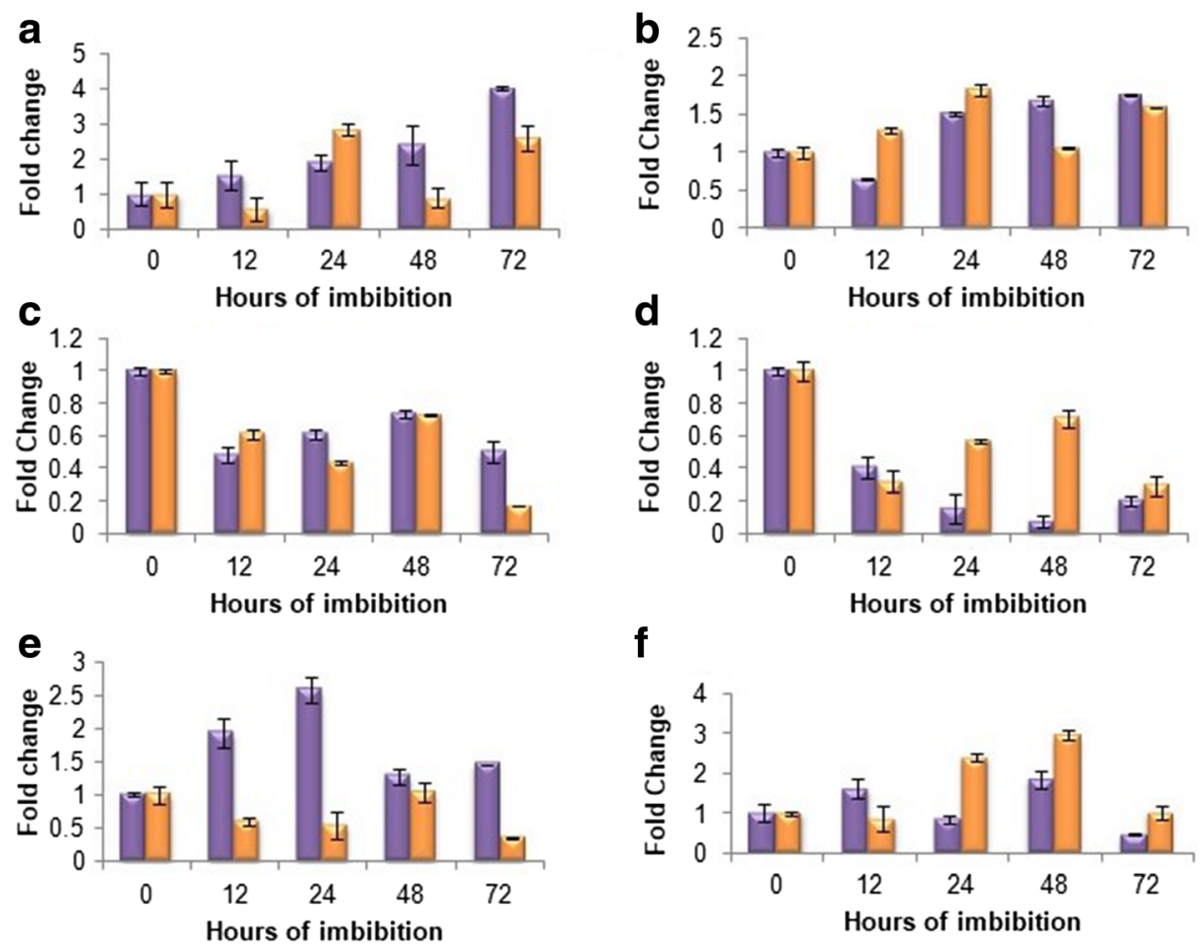

$\mathbf{f}$
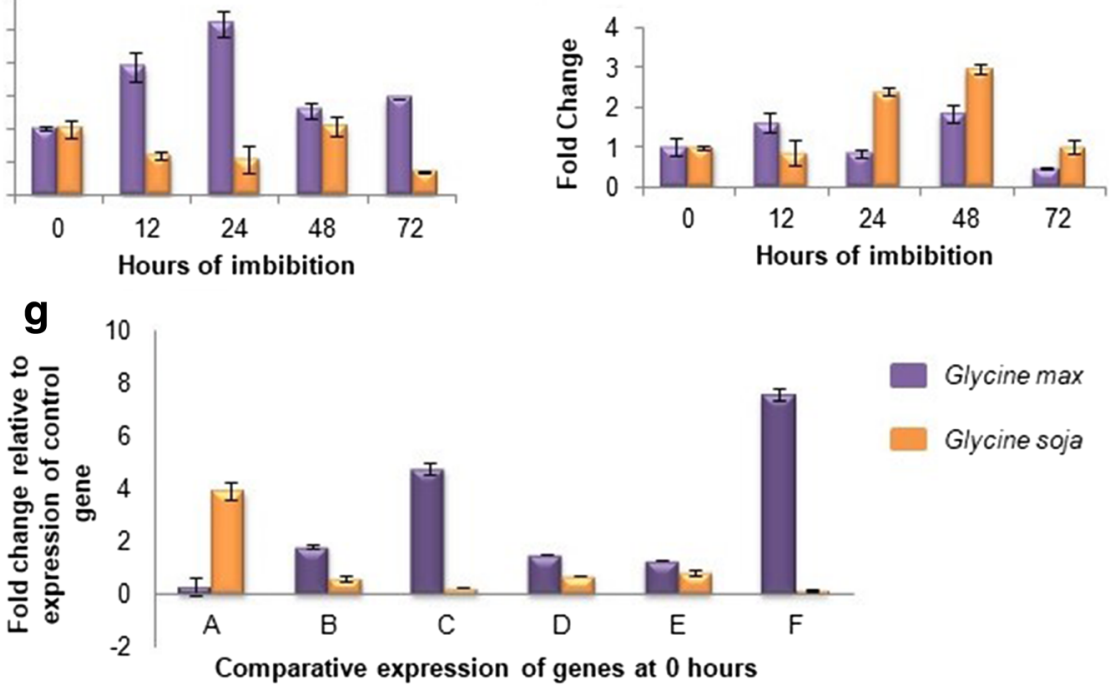

Glycine max

Glycine soja

Fig. 4 Real-time expression studies of 6 genes in G. max and G. soja at 0, 12, 24, 48 and 72 h of imbibition along with computed standard errors (a: Glycosyl transferases; b: Phospholipase D; $\mathbf{c}$ : Anthranilate n-hydroxy cinnamoyl/benzoyl transferase; d: Abscisic acid insensitive 5, e: Chalcone flavone isomerase; $\mathbf{f}$ : E3 Ubiquitin ligase; $\mathbf{g}$ : Comparative expression of genes A-F at $0 \mathrm{~h}$ in $\mathrm{G}$. max and G. soja normalized to the expression data of 18 s rRNA gene)

In silico characterization of effect of variation on amino acid composition and protein structure

Interestingly, for genes Type I/Type I/InsP 5-ptase and E3 Ubiquitin ligase, the respective InDels accounted for an expected segregation pattern among RILs i.e., RILs with a permeability score of 75-100 displayed band pattern similar to G. $\max$ while RILs with permeability score $<10$ depicted band pattern characteristic of G. soja (Fig. 5) whereas for another 5 genes, segregation observed among RILs did not reveal any correspondence with the permeability score.

Amino acid sequences of protein Type I/InsP 5-ptase and E3 Ubiquitin ligase of G. $\max$ and G. soja were then used to identify suitable template structures for comparative 3D modeling. A $10 \mathrm{bp}$ insertion in E3 ubiquitin ligase of G. max, caused a gain of a stop codon leading to protein truncation, thus structural deviations in its protein structure relative to G. soja are obvious and expected (Additional file 11: Figure S5, Additional file 12: Table S2). Of particular interest was the impact of another InDel on the protein conformational structure which was identified as a $12 \mathrm{bp}$ in-frame insertion in G. soja. Deletion of 4 amino acids in G. max relative to G. soja (Fig. 6a) might confer important consequences on protein functioning due to observed conformational distortions and deviations between the two (Fig. 6b). Statistics of the protein modeling with respect to different features are given in Table 3. Selected models for Type I/InsP 5-ptase in G. max and G. soja displayed accurate topology as governed by the C - score, expected TM-score, RMSD value as well as stabilization of its stereo-chemical properties. Stability of protein structures was further confirmed 

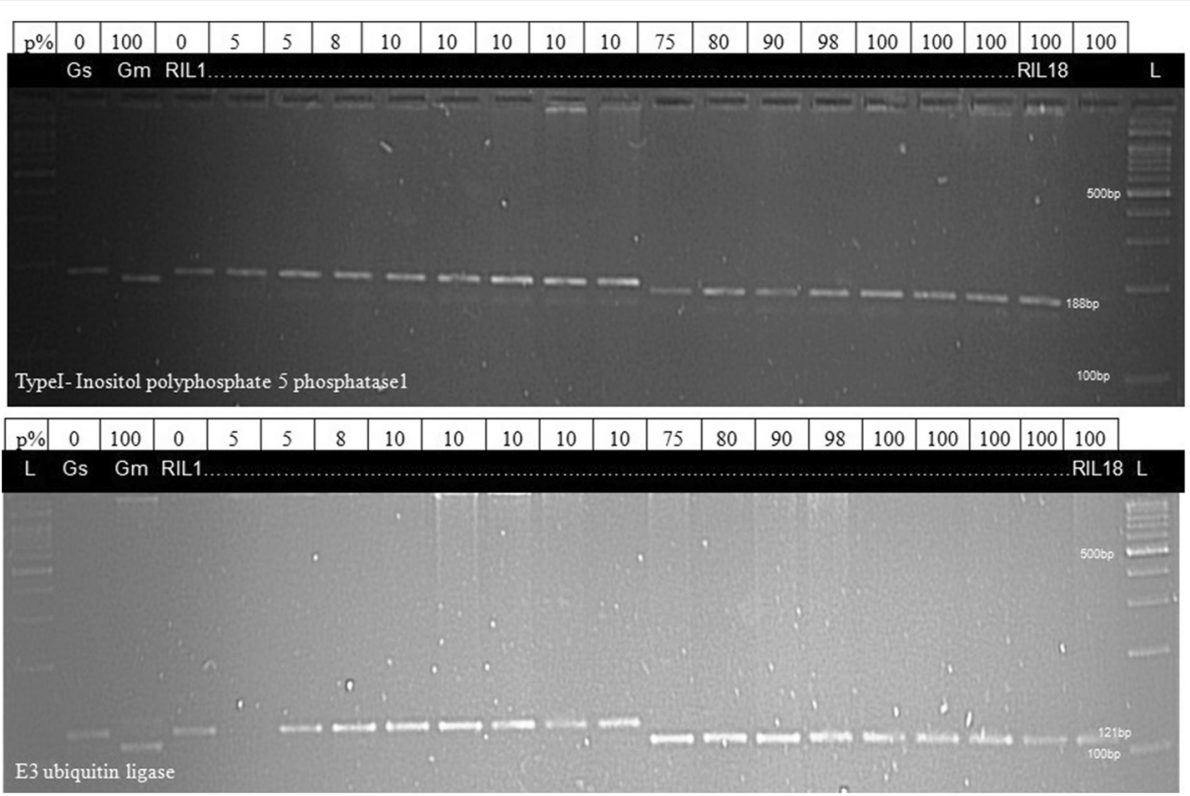

Fig. 5 Segregation pattern observed for genes viz. Typel- Inositol polyphosphate 5 phosphatase1 (top) and E3 ubiquitin ligase (bottom) among 18 RILs (water permeability scores are depicted in a row above the gel view)

\section{a}

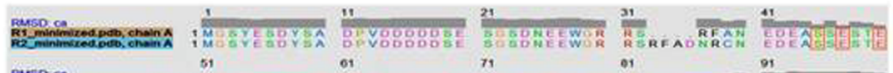

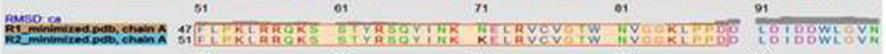

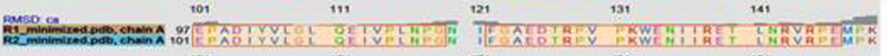

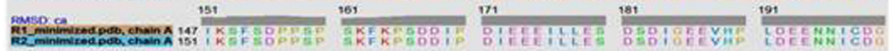

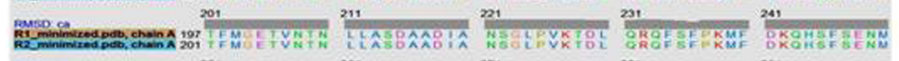

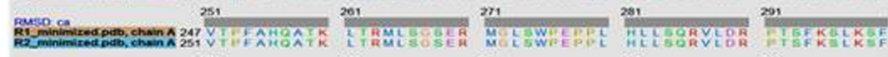

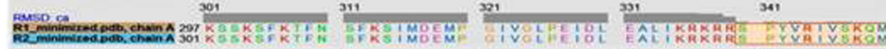

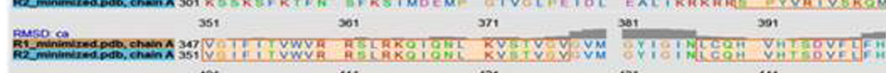

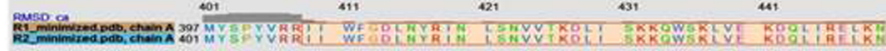

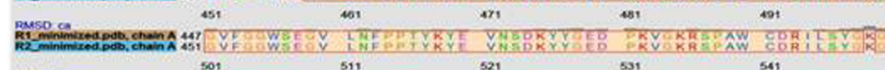
SOT
Ruso S11

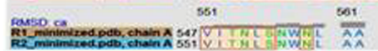

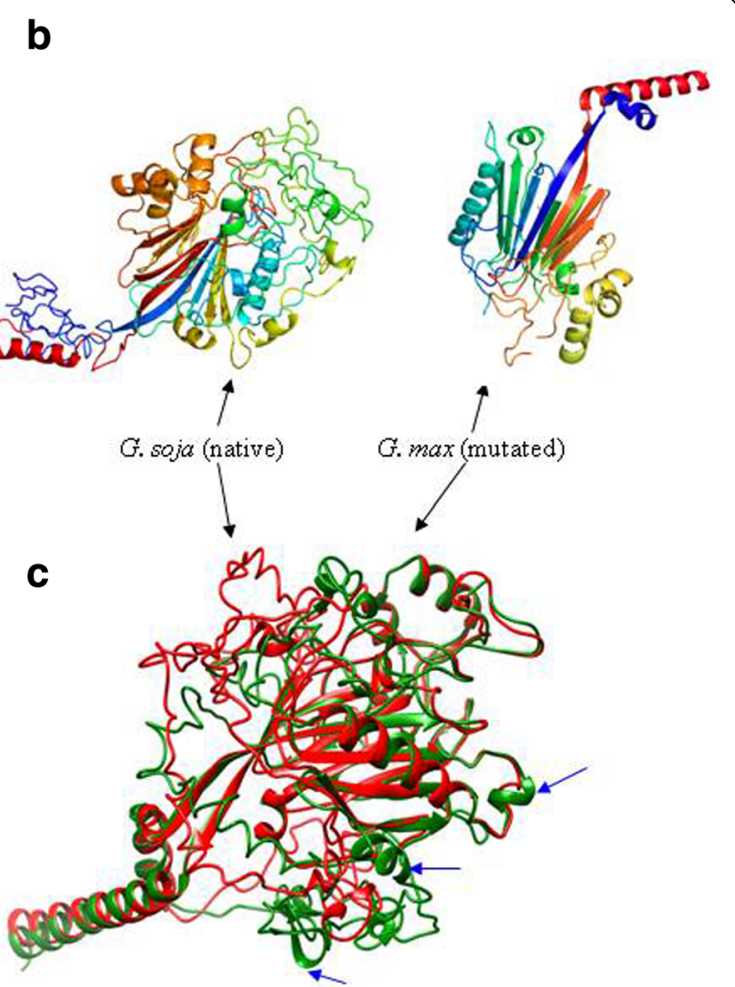

Fig. 6 Pairwise alignment (a) of Typel-Inositol polyphosphate5phosphatase1 protein sequence between G. max and G. soja, (b) 3D structure of protein in G. max and G. soja as predicted by I-TASSER, (c) Structural superimposition of protein through Chimera wherein arrows point out the disordered structure of G. max (R1 and R2 represent G. max and G. soja, respectively) 
Table 3 Statistics of Typel-Inositol polyphosphate5phosphatase1 protein structural predictions in G. max and G. soja (C score: Confidence score, TM score: Template Modeling score, RMSD: root mean square deviation)

\begin{tabular}{|c|c|c|c|}
\hline \multicolumn{2}{|l|}{ Features } & G. $\max$ & G. soja \\
\hline \multicolumn{2}{|c|}{ Top 10 templates predicted by I-TASSER } & $\begin{array}{l}\text { 1i9yA, 1i9yA, 1i9zA, 4cmlA, 4cmnA, 1i9yA, } \\
4 \mathrm{cmlA}, 4 \mathrm{cmnA}, 1 \text { i } 9 y \text { A, } 1 \text { igzA }\end{array}$ & $\begin{array}{l}\text { 1i9yA, 1i9yA, 1i9zA, 4cmnA, 4cmnA, 1i9yA, } \\
\text { 4cmlA, 2xswA, 1i9yA, 1i9zA }\end{array}$ \\
\hline \multirow{5}{*}{$\begin{array}{l}\text { Model Evaluation data of } \\
\text { predicted structures }\end{array}$} & C-score & -2.80 & -2.51 \\
\hline & $\begin{array}{l}\text { Expected TM } \\
\text { score }\end{array}$ & $0.39 \pm 0.13$ & $0.42 \pm 0.14$ \\
\hline & Expected RMSD & $14.5 \pm 3.7$ & $13.8 \pm 3.9$ \\
\hline & $\begin{array}{l}\text { Number of } \\
\text { Decoys }\end{array}$ & 354 & 455 \\
\hline & Cluster Identity & 0.0158 & 0.0210 \\
\hline \multirow[t]{2}{*}{$\begin{array}{l}\text { Energy value }(\mathrm{K} / \mathrm{mole}) \text { of } \\
\text { predicted protein models }\end{array}$} & $\begin{array}{l}\text { before energy } \\
\text { minimization }\end{array}$ & -1148.840 & 190.541 \\
\hline & $\begin{array}{l}\text { after energy } \\
\text { minimization }\end{array}$ & $-17,465.256$ & $-17,325.898$ \\
\hline \multirow{4}{*}{$\begin{array}{l}\text { Ramachandran Plot statistics (\% } \\
\text { residues) }\end{array}$} & Favored regions & 55.9 & 57.3 \\
\hline & Additional region & 31.8 & 30.8 \\
\hline & Allowed regions & 5.9 & 6.9 \\
\hline & $\begin{array}{l}\text { Disallowed } \\
\text { regions }\end{array}$ & 6.3 & 5.1 \\
\hline
\end{tabular}

by Ramachandran plot statistics that revealed the low percentage of amino acid residues to have phi/psi angles in disallowed regions. Further superimposition of refined protein models of $G$. max and G. soja resulted in RMSD value of $0.641 \AA$ and revealed major variations in the secondary structure of the protein, i.e., alpha-helix and loop regions that resulted in overall protein conformational changes from its native to the mutated form (Fig. 6c). These changes might be associated with observed and known permeability differences between G. $\max$ and G. soja seeds. Moreover, global alignment of these proteins at the sequence level also confirmed variation at multiple regions. Hence, Type I/InsP 5-ptase was identified as a novel candidate for determination of seed permeability differences among soybean genotypes but needs further validation at protein function level.

\section{Discussion}

Whole genome resequencing of different soybean accessions has been reported previously to associate SNPs with numerous traits such as biotic stress, domestication, seed composition, seed size, seed coat, flowering etc. [25]. The present study centered on whole-genome resequencing of $G$. $\max$ and $G$. soja to identify variation (SNPs and InDels) present in genes related to seed coat properties with implications on water permeability.

The number of SNPs and InDels identified in the current study are less than those reported earlier due to the high stringency filters used to rule out the possibility of detection of false positive variants and could also be atributed to depth of sequencing. Among soybean cultivars, insertions and deletions of length 1 to $65 \mathrm{bp}$ and 1 to $37 \mathrm{bp}$, respectively [26] to as high as $500 \mathrm{bp}$ [27] has been reported. The observed average density, i.e., the number of SNPs/Mb is identified to be less while InDels/Mb are observed to be more frequent than those in earlier reports [26]. Mapping of maximum SNPs and InDels on chromosome 18 of soybean cultivars with contrasting mungbean yellow mosaic India virus resistance traits has been earlier reported [26] and is consistent with variation distribution observed for G. $\max$ in the present study. Further, SNPs identified were found to be under the influence of transition biases as expected $[28,29]$ with Ts/Tv ratios comparable to previous studies [26] indicating the correctness of our workflow. A/G and $\mathrm{C} / \mathrm{T}$ transitions have also been reported as the most common pattern of nucleotide substitution in white clover [30] and chickpea [31]. A higher frequency of $A / G$ transition was also evident in Desi and Kabuli chickpea [32]. Higher occurrence of the $\mathrm{C} / \mathrm{T}$ transitions is most likely reasoned to occur due to 5-methylcytosine deamination at CpG dinucleotides over time [33, 34]. Further, codon degeneracy for an amino acid is known to be highest at position three followed by position one and position two, which is clearly reflected from the results obtained that allowed a number of variations to occur at degenerate positions of the codon [35]. More abundant distribution of variants in URR and DRR regions of genes is in agreement with reduced selection pressure and low sequence conservation experienced by regulatory regions. Further, mapping of G. soja genes identified 
a diverse array of pathways and generated a valuable functional data resource in the form of SNPs and InDels, which could be associated with different traits.

Out of this diverse spectrum, factors that could influence seed coat permeability were sorted on the basis of literature as described briefly below and putative role of seven selected genes in governing seed permeability is shown in Fig. 7. Flavonols, Anthocyanins, and Proanthocyanidins are known to be major pigments of testa and correlation between seed coat pigmentation and its water imbibing ability has already been demonstrated in common bean [36], faba bean [37], yardlong bean [38] and guar [39]. Additionally, differentially expressed genes (DEGs) identified between seed coat transcriptome of domesticated pea and its wild progenitor revealed that a majority of them function in the phenylpropanoid pathway followed by flavonoid biosynthetic pathways [40]. Further, glycosylation of flavonoids by UGTs (UDP: glucose: flavonoid3-O-glucosyltransferases) is crucial as its Arabidopsis mutants display reduced seed suberization, cuticle formation and show defect in cellulose biosynthesis, and overall affecting seed permeability [41]. Similarly, BAHD acyltransferases mutant exhibits defective cuticle [42] while GPAT5 acyltransferases mutants are characterized with a reduced amount of suberin aliphatic monomers in Arabidopsis seeds, thereby increasing its permeability [12]. Further, ABI5 is known to negatively regulate seed germination [43-45] while PLD (Phospholipase D) is involved in the expression of the GAmyb and $\alpha$-amylase gene in aleurone layer of barley seeds [46]. Moreover, CER9 [47] and PROTEOLYSIS6 [48] genes of Arabidopsis encode for E3 ubiquitin ligase and functions in cuticle biosynthesis as well as negatively regulates $\mathrm{ABA}$ signaling. Another important component of ABA signaling is InsP 5-ptase, whose mutants exhibit increased ABA sensitivity and faster seed germination. This thus justified the selection of 7 genes that were studied further for their role in governing seed permeability in soybean.

Non-synonymous variation can have several functional impacts due to an altered amino acid sequence that could be manifested in several ways, for example, hampering of interaction between proteins. Thus, to assess their role in influencing gene expression, the allele-specific differential binding effect of non-synonymous variant i.e. binding of a particular motif to a specific allele but not to other was analyzed. Ten TF motifs identified to be present uniquely in G. soja laid implications of these onto seed permeability, as reported in previous studies, for example, AP2 is linked to seed dormancy, germination, and longevity in Arabidopsis [49] Similarly, drought responsive Myb transcription factors are associated with the cuticle biosynthesis in legumes [50], which is an important determinant of permeability factor and higher expression of $\mathrm{Myb} /$ SANT domain-containing protein i.e. AtSM34, was

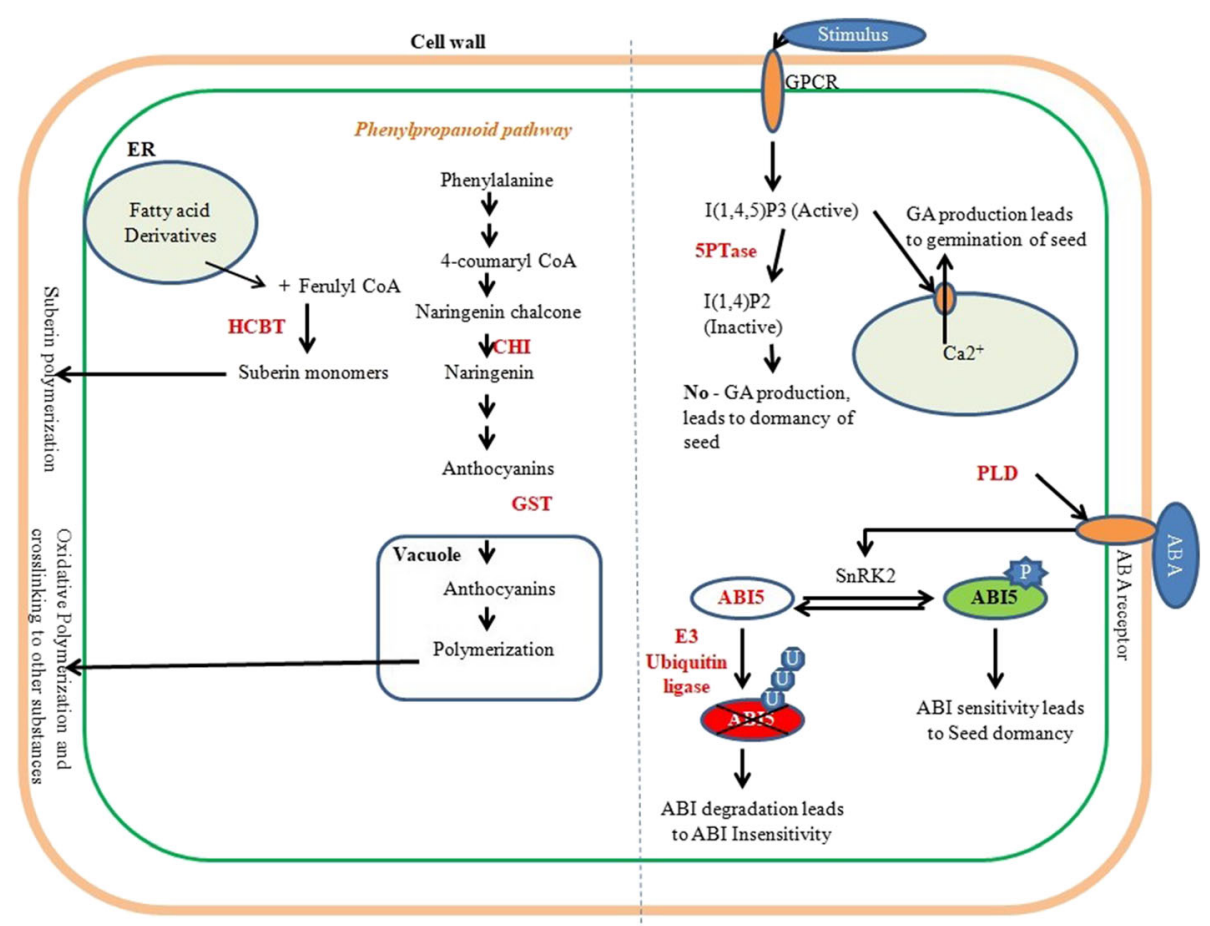

Fig. 7 Hypothetical model depicting the role of action of seven selected genes in determining seed coat permeability properties (HCBT: Anthranilate n-hydroxy cinnamoyl/benzoyl transferase, CHI: Chalcone flavone isomerase, GST: UDP:glucose:flavonoid3-O-glucosyltransferases, InsP 5-ptase: Typel- Inositol polyphosphate 5 phosphatase1, PLD: Phospholipase D and ABI5: Abscisic acid insensitive 5) 
correlated with Arabidopsis seed germination [51]. Homeobox transcription factors are also important determinants of seed longevity [52]. Thus, with currently available analytical tools, further analysis of these transcription factors in association with seed coat permeability is crucial to decipher the underlying mechanism. Downstream interacting partners of 7 selected genes identified through the variant interaction network and their functional annotation, further specified their role in several seed specific processes, particularly permeability. A similar approach has been used to associate 28 deleterious SNPs identified at a genomic scale with genes involved in plant-pathogen interaction and plant hormone pathways for trait targeting in tomato [53].

Non-synonymous variation can make an impact on the gene expression due to the functional consequences of differential motif binding at variation sites [53]. Gene expression studies of selected genes further supported the hypothesis of their direct or indirect involvement in controlling soybean seed permeability. Transcript abundance of $\mathrm{CHI}$ gene at different hours of imbibition in $G$. $\max$ and G. soja seeds correlated well with its role in the production of flavonoids to regulate water movement across the seed coat. Similarly, genes involved in phenyl proponoid pathway and glycosyl transferases exhibit 2 fold up-regulation in the seed coat of black soybean in comparison to the brown seed coat [54]. Upregulation of UDP-glucosyl transferase expression in cultivated pea relative to its wild progenitor [40] is in coherence with the current study. More abundant ABI5 expression in dormant sorghum cultivars [55] as well as in wild, desiccating and dry seeds of Arabidopsis relative to its vegetative tissues has been reported [56]. Similar to the current findings, TaABF, an orthologue of ABI5 in wheat undergoes a reduction in transcript abundance during imbibition of non-dormant seeds in comparison to the transient increase experienced by dormant grains [57]. Lastly, PLD inhibitors are reported to induce the altered emergence of radicle and cotyledons causing inhibition of seed germination $[58,59]$.

Further, marker development from SNPs/InDels identified from sequencing data has been done successfully for various agronomical important traits. InDels identified in gene Type 1/InsP5-ptase (12 bp) and E3 Ubiquitin ligase $(10 \mathrm{bp})$ in the current study also revealed segregation in a RIL population in correlation with the permeability scores of a particular RIL and the banding pattern characteristic for G. max or G. soja. This revealed a preliminary, but a relevant association of these InDels with observed seed permeability differences between cultivated soybean and its wild progenitor and thus enhanced the significance of the present study that could be used to develop simple and efficient PCR and gel-based molecular markers.
In a previously reported study, 22 InDel markers (56-432 bp) were developed in rice to reliably distinguish all genome types of the genus Oryza and were found useful for maintenance of germplasm stocks [60], 2 InDel markers to distinguish rice varieties with $L g c 1$ gene [61] and yield-related functional genes [62] has been reported. Comparative analysis of 4 accessions of chickpea revealed 21,499 genome-wide InDels of length 2-54 bp and identified 5 InDel marker containing candidate genes linked to flowering and maturity time QTLs [63]. In tomato, 2272 polymorphic InDels (1-94 bp) were evaluated in 22 tomato lines to assist in gene cloning and marker-assisted selection [64]. In soybean, InDel markers were used to fine map crinkly leaf locus to a $360 \mathrm{~Kb}$ region on chromosome 7 [65]. Further, Genome-wide analysis of 106 soybean genomes representing the wild, landraces, and elite lines was conducted to discover variation and to associate this variation (SNPs and InDels) with agronomical important and domestication-related traits and for identification of novel alleles [66]. This demonstrates the practical utility of developing InDel markers to expedite genomics-assisted breeding applications.

InDels could have more impact on protein structure and function than single base changes [67], thus allowing their use for development of phylogenetic markers. The evolutionary patterns of InDels from 35 eukaryotic proteomes have recently been studied, including model crop plants, i.e., Arabidopsis and rice [68]. Additionally, protein conformational changes due to InDels resulting in major trait differences in mitochondrial genes are well known. Further, InDels can have a more intense impact on the overall protein structure if they occur within $\alpha$-helices and $ß$-sheets while loops and turns are known to accommodate InDels comparatively well [69]. The observed protein conformational differences at the secondary level for InsP5-ptase due to deletion present at DNA level in G. max relative to G. soja thus might be responsible for their observed differences in seed permeability. Thus, InDels especially those altering protein structures and function have the potential to improve our understanding of the consequence of observed natural variation in a better way.

\section{Conclusion}

The present study is a comprehensive report on the comparison of the genomes of Glycine max and its wild progenitor (G. soja) to decipher and annotate the potential genic variation in an effort to understand seed permeability as evidenced by transcript abundance of genes and water permeability scores. In summary, two candidate genes were identified that 
hold the potential to be associated with differentiating permeability abilities of soybean seeds. Elucidated genomic information, thus provides a valuable resource to facilitate trait dissection based on detection of sequence-based variation with implications for molecular breeding.

\section{Methods}

\section{Wet lab analysis}

\section{Plant material and DNA isolation}

Soybean genotypes with contrasting seed permeability traits were selected, i.e., G. soja accession DC2008-1 (hard seed) and G. $\max$ accession DS9712 (soft seed). Plant material (Leaf and seed samples) was procured from Division of Genetics, IARI, Pusa Campus, New Delhi. Genomic DNA was isolated from leaves of parental genotypes and300RILs (F9) derived from their cross using the standard CTAB method [70] and DNAsure Plant Mini Kit (Nucleo-pore). 18 RILs (F8 generation) were randomly selected on the basis of water permeability scores (unpublished data) i.e., 9 RILs with a score in the range of $0-10$ and other 9 RILs with a score in the range of 75-100 for further analysis. For permeability tests, 20 seeds each of 160 RILs, G. soja accession DC2008-1 and G. max accession DS9712, in triplicates were soaked in $100 \mathrm{ml}$ of distilled water for $6 \mathrm{~h}$ at room temperature $\left(25^{\circ} \mathrm{C}\right)$ as per modified Zhang et al. [21]. After $6 \mathrm{~h}$, seeds were checked for imbibition. The seeds that had not imbibed water were counted as "impermeable or hard seeds" while the seeds that had imbibed water were counted as "permeable or soft seeds". Seed coat permeability was defined as the percentage of soft seeds that had imbibed water after soaking for $6 \mathrm{~h}$. Additional file 13: Figure S6 depict the seeds of G. soja and G. $\max$ before and after imbibition.

\section{Whole genome resequencing}

Genomic DNA from 25 days-old seedlings of G. soja and G. max was isolated using the DNAsure Plant Mini Kit (Nucleo-pore) and its quality and quantity were ascertained by Bioanalyzer 2100 (Agilent Technologies). DNA library was prepared using TruSeq DNA PCR-Free HT Sample Preparation Kit, following the manufacturer's instructions and was processed for paired-end sequencing using the Illumina HiSeq 1000 platform (Illumina Technologies).

\section{Primer designing and DNA polymorphism studies of candidate genes}

Primer pairs targeting the variation in 7 selected genes were designed using an IDT PrimerQuest tool (https:// eu.idtdna.com/PrimerQuest/Home/Index) with amplicon size $\leq 500 \mathrm{bp}$. Primer pairs were custom synthesized through the facility of Eurofins Analytical Services, India
Private Ltd. and were validated using genomic DNA of G. max and G. soja. PCR was performed in 20- $\mu$ l volume in Takara Thermocycler. Reaction mixture contained $0.5 \mathrm{X}$ buffer, $5 \mu \mathrm{M}$ forward + reverse primer, $1.5 \mathrm{mM}$ $\mathrm{MgCl}_{2}, 0.8 \mathrm{mM}$ dNTPs, 1 unit of Taq DNA polymerases (New England Biolabs) and 100 ng of genomic DNA. PCR amplification conditions were: $95{ }^{\circ} \mathrm{C}$ for $5 \mathrm{~min}$, followed by 40 cycles of $95{ }^{\circ} \mathrm{C}$ for $30 \mathrm{Sec}, \mathrm{Tm}\left({ }^{\circ} \mathrm{C}\right)$ for 30 Sec and $72{ }^{\circ} \mathrm{C}$ for $30 \mathrm{Sec}$ and a final step of extension at $72{ }^{\circ} \mathrm{C}$ for $5 \mathrm{~min}$. After amplification, $2 \mu \mathrm{l}$ of $6 \mathrm{X}$ loading dye was added to each of the amplified products which were then resolved in 4\% Metaphor-Agarose gels (TBE buffer) supplemented with ethidium bromide. Sequences of primer pairs designed are listed in Additional file 14: Table S3.

\section{$R N A$ isolation and reverse transcription quantitative $P C R$ (RT-qPCR) analysis}

RNA extraction was performed on 2 replicates of 5 seeds of G. $\max$ and G. soja each at 5 different time intervals, i.e., 0, 12, 24, 48 and $72 \mathrm{~h}$ of imbibition using RaFlex ${ }^{\mathrm{Tm}}$ total RNA Isolation kit (Merck Millipore) as per manufacturer's protocol. RNA quantity and purity was ascertained using NANODROP 2000 (Thermo Scientific) and formamide denaturing gel electrophoresis.For RT-qPCR, $1 \mu \mathrm{g}$ of total RNA was reverse-transcribed using RevertAid First strand cDNA synthesis kit (Thermo Scientific) according to the manufacturer's instructions. First strand cDNA was further diluted to 1:10 ratio for real-time expression studies performed on Roche Light Cycler 480 using SyBr GreenER qPCR Supermix (Invitrogen). Gene expressions from each cDNA sample were normalized to the endogenous reference gene i.e., 18SrRNA. Forward and reverse primers used for RT-qPCR analysis are listed in Additional file 14: Table S3. Relative expression levels were calculated using the comparative $2 \Delta(\mathrm{Ct})$ method [71] and standard errors were calculated.

\section{Bioinformatics analysis \\ Read mapping and discovery of SNPs and InDels}

Initial quality control and cleaning of reads were done using FastQC (http://www.bioinformatics.babraham.ac.uk/ projects/fastqc/) Trimmomatic software at default parameters. High-quality Illumina reads of wild and cultivated soybean were mapped to the reference genome of G. $\max$ (var. Williams 82, ftp://ftp.ensemblgenomes.org/pub/plants/release-35/fasta/glycine_max/dna) utilizing Burrows-Wheeler Aligner (BWA) software (v0.7.10). Samtools and Picard (http://broadinstitute.github.io/picard/) were used to refine the mapping output of BWA, which was further filtered at mapping quality $(M Q \geq 20)$ and base quality $(Q \geq 30)$ for downstream analysis. Genome-wide SNPs and InDels calling was done using Genome Analysis Toolkit (GATK, v3.1.1) software (https://software.broadinstitute.org/gatk/) 
and Samtools/bcftools (https://samtools.github.io/bcftools/) at a minimum read depth of 10 and SNP quality 30 . The Realigner Target Creator and InDels-Realinger features of GATK were used to realign InDels. To further rule out identification of false positives, following criteria were used: (cluster size 3, cluster window size 10 , and filter $\mathrm{DP}<10$, Filter MQ $0>=4\left(\mathrm{MQ} 0 /\left(1.0^{*} \mathrm{DP}\right)>0.1\right)$.

\section{Chromosomal distribution of SNPS}

Genomic distribution and annotation of the SNP and InDels were analyzed using in-house Perl scripts and visualized with Circos software (http://circos.ca/software/). To assess their distribution, variant positions were integrated with a GFF3 file containing annotation data of G. $\max$ (ftp://ftp.ensemblgenomes.org/pub/plants/release-35/gff3/ glycine_max/).

\section{Functional annotation and categorization of SNPs and InDels}

Genes carrying variation were annotated into KEGG pathways with KeggMapper (http://www.genome.jp/ kegg/mapper.html) using G. $\max$ as a reference. Synonymous/ Non-synonymous SNPs and impact of variation in gene function were evaluated using Variant effect Predictor software (http://www.ensembl.org/info/ docs/tools/vep/index.html) in reference to G. soja. Further, SIFT22 (subPSEC score $\leq-3$ ) was used to detect deleterious SNPs.

\section{Presumptive effect of deleterious SNPs/ InDels at structure level and interacting network}

Impact of SNPs and InDels in selected genes was analyzed at the sequence level by prediction of differential binding events utilizing 8 mer motif model and $10 \mathrm{bp}$ flanking gene sequences w.r.t. identified variation via CIS-BP web server (http://cisbp.ccbr.utoronto.ca/ TFTools.php). Further, high confidence interacting partners of selected genes were identified using SoyNet (http://www.inetbio.org/soynet/), which gene to gene relationship based on Bayesian statistics to identify candidate genes for the hypothesis in consideration and interaction network was build and visualized using Cytoscape [72]. Gene ontology (GO) analysis of interacting partners was conducted using an AgriGO program (http://bioinfo.cau.edu.cn/agriGO/).

\section{3-D structure prediction, validation, visualization and analysis}

The three-dimensional structure of variant Type IInositol polyphosphate 5 phosphatase 1 genes and E3 Ubiquitin ligase from G. $\max$ and G. soja were predicted by I-TASSER (http://zhanglab.ccmb.med.umich.edu/I-TASSER/). Predicted protein models were subjected to Structural Analysis and Verification Server
(SAVES) (http://services.mbi.ucla.edu/SAVES) for evaluation and quality checking. Refined protein models were visualized and superimposed by Chimera1.11 (https:// www.cgl.ucsf.edu/chimera/).

\section{Additional files}

Additional file 1: Table S1. Statistics of Assembly generated from Illumina HiSeq1000 sequencing data. (DOC 29 kb)

Additional file 2: Identification of total SNPs and InDels in G. max with respect to the reference genome. (XLSX $44647 \mathrm{~kb}$ )

Additional file 3: Identification of total SNPs in G. soja with respect to reference genome. (XLSX $19576 \mathrm{~kb}$ )

Additional file 4: Identification of total InDels in G. soja with respect to reference genome. (CSV $97568 \mathrm{~kb}$ )

Additional file 5: Functional classification of non-synonymous SNPs and InDels into diverse pathways. (XLS 636 kb)

Additional file 6: Figure S1. GO annotation of 205 interacting partners of 7 selected genes into biological processes. (PNG $309 \mathrm{~kb}$ )

Additional file 7: Figure S2. GO annotation of 205 interacting partners of 7 selected genes into molecular functions. (PNG $307 \mathrm{~kb}$ )

Additional file 8: Figure S3. GO annotation of 205 interacting partners of 7 selected genes into cellular compartments. (PNG $31 \mathrm{~kb}$ )

Additional file 9: Functional annotation of 205 interacting patterns as identified for 7 selected genes. (XLS $184 \mathrm{~kb}$ )

Additional file 10: Figure S4. DNA polymorphism analysis of genes in G. max (Gm) and G. soja (Gs) in order Anthranilate n-hydroxy cinnamoyl/ benzoyl transferase, Chalcone flavone isomerase, Abscisic acid insensitive 5, Phospholipase D, Typel- Inositol polyphosphate 5 phosphatase1, E3 Ubiquitin ligase and Glycosyltransferase. (JPG $111 \mathrm{~kb}$ )

Additional file 11: Figure S5. Pairwise alignment (a) of protein sequence of E3 Ubiquitin ligase from G. max and G. soja, (b) 3D structure of protein in G. max and G. soja as predicted by I-TASSER, (c) Structural superimposition of protein obtained through Chimera ( $\mathrm{Gm}$ and Gs represent G. max and G. soja, respectively). (JPG 154 kb)

Additional file 12: Table S2. Statistics of E3 Ubiquitin ligase protein structural predictions in G. max and G. soja (TM score: Template Modeling score, RMSD: root mean square deviation). (DOC $34 \mathrm{~kb}$ )

Additional file 13: Figure S6. Seeds of G. soja and G. max before (Control seeds, 0 h) and after water imbibition of 6 h. (JPG 7 kb)

Additional file 14: Table S3. List of forward and reverse primers designed for detection of DNA polymorphism and real-time analysis for selected genes. (DOC $30 \mathrm{~kb}$ )

\section{Abbreviations}

ABA: Abscisic acid; ABI: Abscisic acid insensitive; InDels: Insertion deletions; RFLP: Restriction fragment length polymorphism; RIL: Recombinant inbred line; SNP: Single nucleotide polymorphism; SSR: Simple sequence repeat

\section{Acknowledgements}

The authors are grateful to the Director, National Research Centre on Plant Biotechnology (NRCPB), New Delhi, India for providing facilities. The authors also thank the other members of our research groups and collaborators for technical assistance and discussions.

\section{Funding}

This work is supported by the ICAR- National Agricultural Science Fund, New Delhi, India. The role of the funding body is to provide financial support and advise/guide the team as and when required. This is done by mid term and annual review of the progress of the project. In the current study, design, collection, data analysis, data interpretation and manuscript writing was done exclusively by the authors as mentioned in the author contribution section. 


\section{Availability of data and materials}

All the genomic sequence information generated in the current study has been deposited in a BioProject database of NCBI, under ID PRJNA383915. All other data associated with the current study are available from the corresponding author on reasonable request.

\section{Authors' contributions}

KG, GR, PK designed and executed the experiments. PK, DN, and GR wrote the manuscript. DN and PC carried out the Bioinformatics work. SY, AT and NKS provided inputs, material and helped in manuscript writing. All authors have critically read and approved the final manuscript.

\section{Ethics approval and consent to participate}

Not applicable.

\section{Competing interests}

The authors declare that they have no competing interests.

\section{Publisher's Note}

Springer Nature remains neutral with regard to jurisdictional claims in published maps and institutional affiliations.

\section{Author details}

'ICAR- National Research Centre on Plant Biotechnology, Pusa Campus, New Delhi 110012, India. ${ }^{2}$ ICAR- IARI, Division of Seed Science and Technology, Pusa Campus, New Delhi 110012, India. ${ }^{3}$ ICAR- IARI, Division of Genetics, Pusa Campus, New Delhi, India.

Received: 13 September 2017 Accepted: 5 June 2018

Published online: 09 July 2018

\section{References}

1. Ragus $L N$. Role of water absorbing capacity in soybean germination and seedling vigor. Seed Sci Technol. 1987;15:285-96.

2. Chachalis D, Smith ML. Imbibition behavior of soybean (Glycine max (L.) Merrill) accessions with different testa characteristics. Seed Sci Technol. 2000;28:321-31.

3. Mullin WJ, Xu W. Study of soybean seed coat components and their relationship to water absorption. J Agric Food Chem. 2001;49:5331-5.

4. Tyler JM. Effect of impermeable seed coat on germination of seed from early maturing soybean. Seed Technol. 1997:45-50.

5. Jamil Y, Ahmad MR. Effect of pre-sowing magnetic field treatment to garden pea (Pisum sativum L.) seed on germination and seedling growth. Pak J Bot. 2012:44:1851-6.

6. Ma F, Cholewa E, Mohamed T, Peterson CA, Gijzen M. Cracks in the palisade cuticle of soybean seed coats correlate with their permeability to water. Ann Bot. 2004:94:213-28

7. Zhou S, Sekizaki H, Yang Z, Sawa S, Pan J. Phenolics in the seed coat of wild soybean (Glycine soja) and their significance for seed hardness and seed germination. J Agric Food Chem. 2010;58:10972-8.

8. Panikashvili D, Shi JX, Schreiber L, Aharoni A. The Arabidopsis DCR encoding a soluble BAHD acyltransferase is required for cutin polyester formation and seed hydration properties. Plant Physiol. 2009;151:1773-89.

9. Molina I, Ohlrogge JB, Pollard M. Deposition and localization of lipid polyester in developing seeds of Brassica napus and Arabidopsis thaliana. Plant J. 2008:53:437-49.

10. Ranathunge K, Shao S, Qutob D, Gijzen M, Peterson CA, Bernards MA. Properties of the soybean seed coat cuticle change during development. Planta. 2010;231:1171-88.

11. Liang M, Davis E, Gardner D, Cai X, Wu Y. Involvement of AtLAC15 in lignin synthesis in seeds and in root elongation of Arabidopsis. Planta. 2006;224:1185-96.

12. Beisson F, Li Y, Bonaventure G, Pollard M, Ohlrogge JB. The acyltransferase GPAT5 is required for the synthesis of suberin in seed coat and root of Arabidopsis. Plant Cell. 2007:19:351-68.

13. Nonogaki H. Seed dormancy and germination-emerging mechanisms and new hypotheses. Front Plant Sci. 2014:5:233.

14. Yoshida T, Mogami J, Yamaguchi-Shinozaki K. ABA-dependent and ABAindependent signaling in response to osmotic stress in plants. Curr Opin Plant Biol. 2014;21:133-9.
15. Yan D, Duermeyer $L$, Leoveanu C, Nambara $E$. The functions of the endosperm during seed germination. Plant Cell Physiol. 2014;55:1521-33.

16. De Giorgi J, Piskurewicz U, Loubery S, Utz-Pugin A, Bailly C, MèneSaffrané $L$, et al. An endosperm-associated cuticle is required for Arabidopsis seed viability, dormancy and early control of germination. PLoS Genet. 2015:11:1-32.

17. Maia J, Dekkers BJW, Dolle MJ, Ligterink W, Hilhorst HWM. Abscisic acid (ABA) sensitivity regulates desiccation tolerance in germinated Arabidopsis seeds. New Phytol. 2014;203:81-93.

18. Terrasson E, Buitink J, Righetti K, Ly Vu B, Pelletier S, Zinsmeister J, et al. An emerging picture of the seed desiccome: confirmed regulators and newcomers identified using transcriptome comparison. Front Plant Sci. 2013:4:497.

19. Keim P, Diers BW, Shoemaker RC. Genetic analysis of soybean hard seededness with molecular markers. Theor Appl Genet. 1990;79:465-9.

20. Watanabe S, Tajuddin T, Yamanaka N, Hayashi M, Harada K. Analysis of QTLs for reproductive development and seed quality traits in soybean using recombinant inbred lines. Breed Sci. 2004;54:399-407.

21. Zhang B, Chen P, Chen CY, Wang D, Shi A, Hou A, et al. Quantitative trait loci mapping of seed hardness in soybean. Crop Sci. 2008;48:1341-9.

22. Liu B, Fujita T, Yan Z-H, Sakamoto S, Xu D, Abe J. QTL mapping of domestication-related traits in soybean (Glycine max). Ann Bot. 2007:100:1027-38

23. Sun L, Miao Z, Cai C, Zhang D, Zhao M, Wu Y, et al. GmHs1-1, encoding a calcineurin-like protein, controls hard-seededness in soybean. Nat Genet. 2015:47:939-43.

24. Jang SJ, Sato M, Sato K, Jitsuyama Y, Fujino K, Mori H, et al. A singlenucleotide polymorphism in an endo-1,4-B-glucanase gene controls seed coat permeability in soybean. PLoS One. 2015;10:1-19.

25. Kumawat G, Gupta S, Ratnaparkhe MB, Maranna S, Satpute GK. QTLomics in soybean: a way forward for translational genomics and breeding. Front Plant Sci. 2016:7:1852.

26. Yadav CB, Bhareti $P$, Muthamilarasan M, Mukherjee M, Khan $Y$, Rathi $P$, et al. Genome-wide SNP identification and characterization in two soybean cultivars with contrasting mungbean yellow mosaic India virus disease resistance traits. PLoS One. 2015:10:e0123897.

27. Yang J, Shi X, Hu L, Luo D, Peng J, Xiong S, et al. InDel marker detection by integration of multiple softwares using machine learning techniques. BMC Bioinfo. 2016:1-11.

28. Riju A, Chandrasekar A, Arunachalam V. Mining for single nucleotide polymorphisms and insertions / deletions in expressed sequence tag libraries of oil palm. Bioinformation. 2007;2:128-31.

29. Chandrasekar A, Riju A, Sithara K, Anoop S, Eapen SJ. Identification of single nucleotide polymorphism in ginger using expressed sequence tags. Bioinformation. 2009:4:119-22.

30. Hand ML, Ponting RC, Drayton MC, Lawless KA, Cogan NOI, Charles

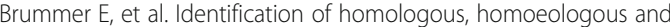
paralogous sequence variants in an outbreeding allopolyploid species based on comparison with progenitor taxa. Mol Gen Genomics. 2008;280:293-304

31. Gaur R, Azam S, Jeena G, Khan AW, Choudhary S, Jain M, et al. High-throughput SNP discovery and genotyping for constructing a saturated linkage map of chickpea (Cicer arietinum L.). DNA Res. 2012;19:357-73

32. Kujur A, Bajaj D, Upadhyaya HD, Das S, Ranjan R, Shree T, et al. Employing genome-wide SNP discovery and genotyping strategy to extrapolate the natural allelic diversity and domestication patterns in chickpea. Front Plant Sci. 2015;6:162.

33. Holliday R, Grigg GW. DNA methylation and mutation. Mutat Res - Fundam Mol Mech Mutagen. 1993;285:61-7.

34. Leonforte A, Sudheesh S, Cogan NO, Salisbury PA, Nicolas ME, Materne M, et al. SNP marker discovery, linkage map construction and identification of QTLs for enhanced salinity tolerance in field pea (Pisum sativum L.). BMC Plant Biol. 2013:13:161.

35. Castle JC. SNPS occur in regions with less genomic sequence conservation. PLoS One. 2011;6:e20660.

36. Caldas GV, Blair MW. Inheritance of seed condensed tannins and their relationship with seed-coat color and pattern genes in common bean (Phaseolus vulgaris L.). Theor Appl Genet. 2009;119:131-42.

37. Ramsay G. Inheritance and linkage of a gene for testa-imposed seed dormancy in faba bean (Vicia faba L.). Plant Breed. 1997;116:287-9. 
38. Kongjaimun A, Kaga A, Tomooka N, Somta P, Vaughan DA, Srinives P. The genetics of domestication of yardlong bean, Vigna unguiculata (L.) Walp. Ssp. unguiculata CV.-gr. Sesquipedalis. Ann Bot. 2012;109:1185-200.

39. Liu W, Peffley EB, Powell RJ, Auld DL, Hou A. Association of seedcoat color with seed water uptake, germination, and seed components in guar (Cyamopsis tetragonoloba (L.) Taub). J Arid Environ. 2007;70:29-38.

40. Smýkal $P$, Hradilová I, Trněný $O$, Válková $M$, Cechová $M$, Janská $A$, et al. $A$ combined comparative transcriptomic, Metabolomic, and anatomical analyses of two key domestication traits: pod dehiscence and seed dormancy in pea (Pisum sp.). Front Plant Sci. 2017;8:542.

41. DeBolt S, Scheible W-R, Schrick K, Auer M, Beisson F, Bischoff V, et al. Mutations in UDP-glucose:sterol glucosyltransferase in Arabidopsis cause transparent Testa phenotype and Suberization defect in seeds. Plant Physiol. 2009;151:78-87.

42. Wu L, Zhou ZY, Zhang CG, Chai J, Zhou Q, Wang L, et al. Functional roles of three cutin biosynthetic acyltransferases in cytokinin responses and skotomorphogenesis. PLoS One. 2015;10:1-21.

43. Piskurewicz U, Jikumaru Y, Kinoshita N, Nambara E, Kamiya Y, Lopez-Molina $L$. The gibberellic acid signaling repressor RGL2 inhibits Arabidopsis seed germination by stimulating abscisic acid synthesis and ABI5 activity. Plant Cell. 2008;20:2729-45.

44. Kanai M, Nishimura M, Hayashi M. A peroxisomal ABC transporter promotes seed germination by inducing pectin degradation under the control of ABI5. Plant J 2010;62:936

45. Zinsmeister J, Lalanne D, Terrasson E, Chatelain E, Vandecasteele C, Vu BL, Dubois-Laurent C, Geoffriau E, Le Signor C, Dalmais M, Gutbrod K, Dörmann P, Gallardo K, Bendahmane A, Buitink J, Leprince O. ABI5 is a regulator of seed maturation and longevity in legumes. Plant Cell. 2016;28(11):2735-54.

46. Shen Q, Gomez-Cadenas A, Zhang P, Walker-Simmons MK, Sheen J, Ho TH. Dissection of abscisic acid signal transduction pathways in barley aleurone layers. Plant Mol Biol. 2001;47:437-48.

47. Zhao $H$, Zhang $H$, Cui P, Ding F, Wang G, Li R, et al. The putative E3 ubiquitin ligase ECERIFERUM9 regulates abscisic acid biosynthesis and response during seed germination and Postgermination growth in Arabidopsis1[W][OPEN]. Plant Physiol. 2014;165:1255-68.

48. Holman TJ, Jones PD, Russell L, Medhurst A, Ubeda Tomas S, Talloji P, et al. The $\mathrm{N}$-end rule pathway promotes seed germination and establishment through removal of ABA sensitivity in Arabidopsis. Proc Nat Acad Sci. 2009;106:4549-54.

49. Wang CM, Wang HW, Zhang JS, Chen SY. A seed-specific AP2-domain transcription factor from soybean plays a certain role in regulation of seed germination. Science in China, series C. Life Sci. 2008;51:336-45.

50. Smýkal P, Vernoud V, Blair MW, Soukup A, Thompson RD. The role of the testa during development and in establishment of dormancy of the legume seed. Front Plant Sci. 2014;5:351.

51. Feller A, MacHemer K, Braun EL, Grotewold E. Evolutionary and comparative analysis of MYB and bHLH plant transcription factors. Plant J. 2011;66:94-116.

52. Bueso E, Muñoz-Bertomeu J, Campos F, Brunaud V, Martínez L, Sayas E, et al. Arabidopsis thaliana HOMEOBOX25 uncovers a role for gibberellins in seed Longevity1[C][W]. Plant Physiol. 2014;164:999-1010.

53. Bhardwaj A, Dhar YV, Asif MH, Bag SK. In Silico identification of SNP diversity in cultivated and wild tomato species: insight from molecular simulations. Sci Rep. 2016;6:38715.

54. Kovinich N, Saleem A, Arnason JT, Miki B. Combined analysis of transcriptome and metabolite data reveals extensive differences between black and brown nearly-isogenic soybean (Glycine max) seed coats enabling the identification of pigment isogenes. BMC Genomics. 2011;12:381.

55. Rodríguez MV, Mendiondo GM, Maskin L, Gudesblat GE, lusem ND, BenechArnold RL. Expression of ABA signalling genes and ABI5 protein levels in imbibed Sorghum bicolor caryopses with contrasting dormancy and at different developmental stages. Ann Bot. 2009;104:975-85.

56. Finkelstein RR, Lynch TJ. The Arabidopsis abscisic acid response gene ABI5 encodes a basic leucine zipper transcription factor. Plant Cell. 2000;12:599-609.

57. Johnson RR, Wagner RL, Verhey SD, Walker-Simmons MK. The abscisic acid-responsive kinase PKABA1 interacts with a seed-specific abscisic acid response element-binding factor, TaABF, and phosphorylates TaABF peptide sequences. Plant Physiol. 2002;130:837-46.

58. Ohashi Y. Modulation of phospholipid signaling by GLABRA2 in root-hair pattern formation. Sci. 2003:300:1427-30.
59. Gardiner J, Collings DA, Harper JDI, Marc J. The effects of the phospholipase D-antagonist 1-butanol on seedling development and microtubule organisation in Arabidopsis. Plant Cell Physiol. 2003:44:687-96.

60. Yamaki S, Ohyanagi H, Yamasaki M, Eiguchi M, Miyabayashi T, Kubo T, et al. Development of InDel markers to discriminate all genome types rapidly in the genus Oryza. Breed Sci. 2013;63:246-54.

61. Tian M, Chen T, Zhang Y, Zhu Z, Zhao L, Zhao Q, et al. Design and validation of two InDel markers for low Glutelin content (LgC1) gene in Rice (Oryza sativa L.). Rice Genomics Genet. 2012;3:50-4. Available from: http://biopublisher.ca/index.php/rgg/article/html/448/

62. Kim S-R, Ramos J, Ashikari M, Virk PS, Torres EA, Nissila E, et al. Development and validation of allele-specific SNP/indel markers for eight yield-enhancing genes using whole-genome sequencing strategy to increase yield potential of rice, Oryza sativa L. Rice. 2016;9:12.

63. Das S, Upadhyaya HD, Srivastava R, Bajaj D, Gowda CLL, Sharma S, et al. Genome-wide insertion-deletion (InDel) marker discovery and genotyping for genomics-assisted breeding applications in chickpea. DNA Res. 2015;22:377-86.

64. Yang J, Wang Y, Shen H, Yang W. In Silico identification and experimental validation of insertion-deletion polymorphisms in tomato genome. DNA Res. 2014;21:429-38.

65. Song $X$, Wei H, Cheng W, Yang S, Zhao Y, Li X, et al. Development of InDel markers for genetic mapping based on whole-genome re-sequencing in soybean. Genes Genomes Genet. 2015;5:2793-9.

66. Valliyodan B, Dan Q, Patil G, Zeng P, Huang J, Dai L, et al. Landscape of genomic diversity and trait discovery in soybean. Sci Rep. 2016;6:23598.

67. Rokas A, Holland PWH. Rare genomic changes as a tool for phylogenetics. Trends Ecol Evol. 2017;15:454-9.

68. Ajawatanawong $\mathrm{P}$, Baldauf SL. Evolution of protein indels in plants, animals and fungi. BMC Evol Biol. 2013;13:140.

69. Kim R, Guo J. Systematic analysis of short internal indels and their impact on protein folding. BMC Struct Biol. 2010;10:24.

70. Porebski S, Bailey LG, Baum BR. Modification of a CTAB DNA extraction protocol for plants containing high polysaccharide and polyphenol components. Plant Mol Biol Rep. 1997;15:8-15.

71. Schmittgen TD, Livak KJ. Analyzing real-time PCR data by the comparative CT method. Nat Protoc. 2008;3:1101-8.

72. Shannon P, Markiel A, Ozier O, Baliga NS, Wang JT, Ramage D, et al. Cytoscape: a software environment for integrated models of biomolecular interaction networks. Genome Res. 2003:13:2498.

\section{Ready to submit your research? Choose BMC and benefit from:}

- fast, convenient online submission

- thorough peer review by experienced researchers in your field

- rapid publication on acceptance

- support for research data, including large and complex data types

- gold Open Access which fosters wider collaboration and increased citations

- maximum visibility for your research: over $100 \mathrm{M}$ website views per year

At BMC, research is always in progress.

Learn more biomedcentral.com/submissions 